\title{
The Line Design of the Logistics Network in Underground Logistics System
}

\author{
Sun Yue ${ }^{1, a, ~}$, Qi Shumei ${ }^{2, ~ b}$ and Qi Yingxiu ${ }^{1, c}$ \\ ${ }^{1}$ School of Traffic and Transportation, Beijing Jiaotong University, Beijing 100044, China \\ ${ }^{2}$ School of Information Management, Jiangxi University of Finance and Economics, Nanchang \\ 330033, China

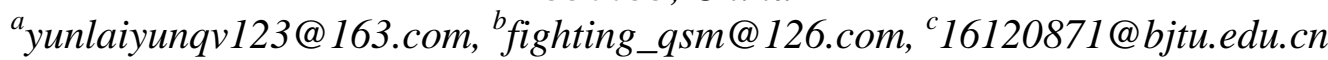

Keywords: Underground logistics system, Fast clustering algorithm based on representative points, Line design.

\begin{abstract}
The main cause of urban traffic congestion is the huge increase in the number of vehicles and trains on the ground road caused by the surge of traffic demand, part of which is the growth of demand for goods logistics. The underground logistics system (Underground Logistics System--ULS) refers to the transportation and supply system for transporting goods within and between cities through underground pipelines or tunnels that resemble subways. The research and practice of ULS has just arisen, there is no mature and successful case for reference. Therefore, the prospective study of urban scale is urgently needed to explore the theory and practice of developing the underground logistics system.
\end{abstract}

\section{Introduction}

This paper studied the line design of ULS, with key points being specified below:

First, the characteristics of line design of ULS were analyzed. Combining the operating characteristics and operating modes of ULS lines, this paper gave detailed explanation of the design principle and influencing factors of ULS lines and set out basic procedures of line design. Second, the demand data for ULS was pre-processed, including the collection and classification of demand and the division of traffic zones. The FCBRP algorithm was applied to the clustering problem of the sites. Third, Routing Design for ULS model was established. The objective functions of the model include three components: the minimization of the traffic congestion and logistics costs. Finally, a calculation sample was designed and MATLAB was used to solve, verify and analyze the algorithm and model. Using the math model and algorithm established above, this paper carried out the line design of ULS, including the clustering of sites and route design. 


\section{Methodology}

\subsection{Selection of regional alternative nodes}

To the center of freight area, 3 kilometers (the service range of the area node) as the radius, draw the Freight Area center circle, think that the region alternative node should choose from the intersection point of the center circle of the freight area. Using MATLAB for data and image processing, the data quantity of the intersection of the center circle of the freight area is 6,530 (duplicates are eliminated). The intersection of Freight Area Center circle and the coordinate distribution of intersection point are presented as shown in Figure 1 (The Freight Regional Center is marked in red), Figure 2 (The Freight Area center point is marked in red, the intersection of the center circle of the freight area is marked in blue).

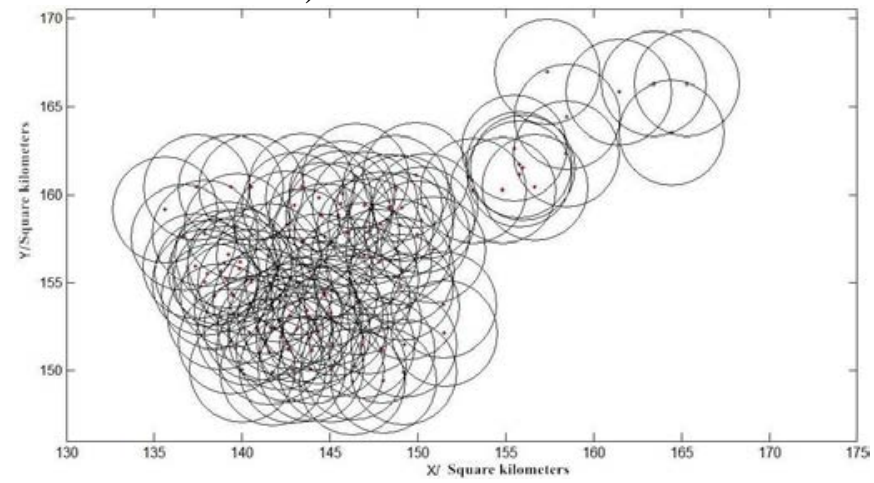

Fig. 1 The intersection of Freight Area Center Circle

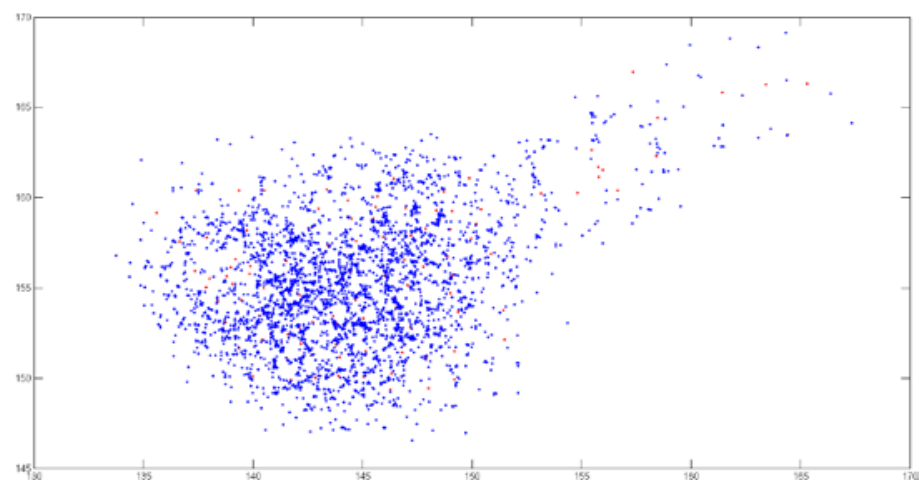

Fig. 2 Coordinate distribution of the nodal solution of the central circle of freight forwarding area

\subsection{Fast clustering algorithm based on representative points}

Using FCBRP (Fast Clustering Based Representative Points) clustering algorithm, cluster analysis the intersection point set of freight Area center circle, and determine 50 region alternative nodes (Fig. 3 ), the region alternative nodes obtained by clustering are marked with large red dots, not similar clusters. With colored dots), the specific process of clustering is:

Create $\mathrm{E}=\varnothing$;

For each $\mathrm{a}_{\mathrm{i}} \in \mathrm{A}$

$$
E\left(a_{i}\right)=0 \text {; }
$$


Is $\mathrm{E}\left(\mathrm{a}_{\mathrm{i}}\right)=0$;

For each $\mathrm{a}_{\mathrm{i}} \in \mathrm{A}$

If $\left(\mathrm{E}\left(\mathrm{a}_{\mathrm{i}}\right) !=1\right.$;

If (Num_of_D $\left(\mathrm{a}_{\mathrm{i}}\right) \geq \mathrm{K}$ )

Is $\mathrm{E}\left(\mathrm{a}_{\mathrm{i}}\right)=1$;

Create $\mathrm{E}+=\mathrm{a}_{\mathrm{i}}$

Create $\mathrm{A}\left(\mathrm{a}_{\mathrm{i}}\right)=\varnothing$;

Relative_D $\left(\mathrm{a}_{\mathrm{i}}\right)=0$;

For each $\mathrm{a}_{\mathrm{i}} \in$ Range of $\mathrm{D}\left(\mathrm{a}_{\mathrm{i}}\right)$;

Create $\mathrm{A}\left(\mathrm{a}_{\mathrm{i}}\right)+=\mathrm{a}_{\mathrm{j}}$

$\operatorname{Be} E\left(a_{j}\right)=1$;

Create $E\left(a_{j}\right)=\varnothing$;

Create $E\left(a_{j}\right)+=a_{i}$;

$a_{i} D\left(a_{i}\right)+=D\left(a_{i}, a_{j}\right) ;$

$\mathrm{D}\left(\mathrm{a}_{\mathrm{i}}\right) /=$ Num_of_D $\left(\mathrm{v}_{\mathrm{i}}\right)$

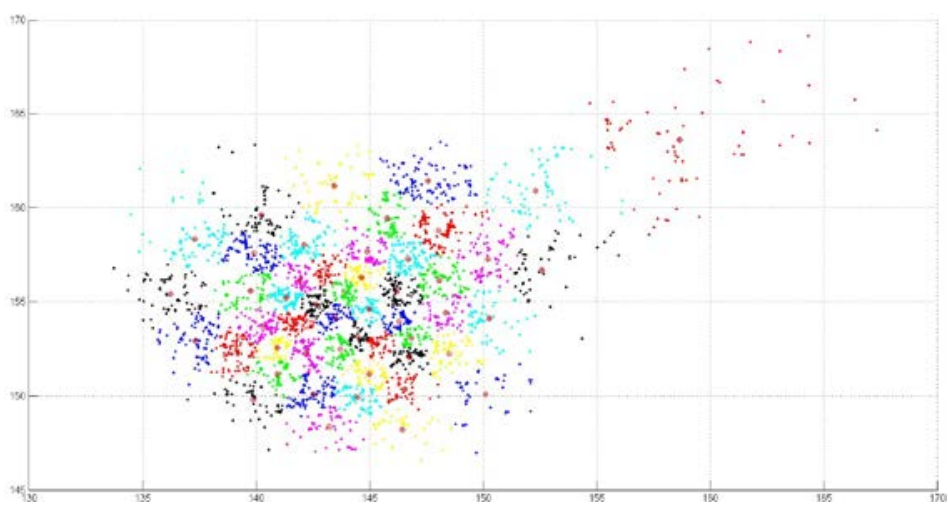

Fig. 3 Clustering of regional alternative nodes

\subsection{Logistics location model}

Build the set overlay model of the regional alternative nodes, the target function is represented as:

$$
\min Z(x)=p_{1} M+p_{2} \sum_{k=1}^{m} \sum_{i=0}^{n} \sum_{j=0}^{n} c_{i j} x_{i j k}+p_{3} \sum_{j=1}^{2 n} \max \left(0, e_{i}-T_{i}\right)+p_{4} \sum_{j \in H} x_{j}
$$

The logistics location model is represented as: 


$$
\begin{gathered}
\sum_{k=1}^{m} \sum_{i=0}^{n} x_{i j k}=1, \forall j \in V \backslash\{0\} \\
\sum_{k=1}^{n} \sum_{i=1}^{n} x_{i 0 k}=\sum_{k=1}^{m} \sum_{j=1}^{n} x_{0 j k}=M \\
\sum_{i \in S} \sum_{j \in \bar{S}} x_{i j k} \geqslant 1, \forall S \subseteq V \backslash\{0\},|S| \geqslant 2, \forall k \in K \\
x_{i j k}=0 \text { 或 } 1, \forall i, j \in V, i \neq j, \forall k \in K
\end{gathered}
$$

\section{Empirical Research}

Using MATLAB to solve the cost minimization model, in order to further meet the cost minimization, the result of the solution is adjusted, the node coordinates numbered 31 are adjusted to (149.5800,154.3200), and the node coordinates numbered 33 are adjusted to $(162.6500,165.8900)$. The adjusted underground channel network is shown in Figure 4.

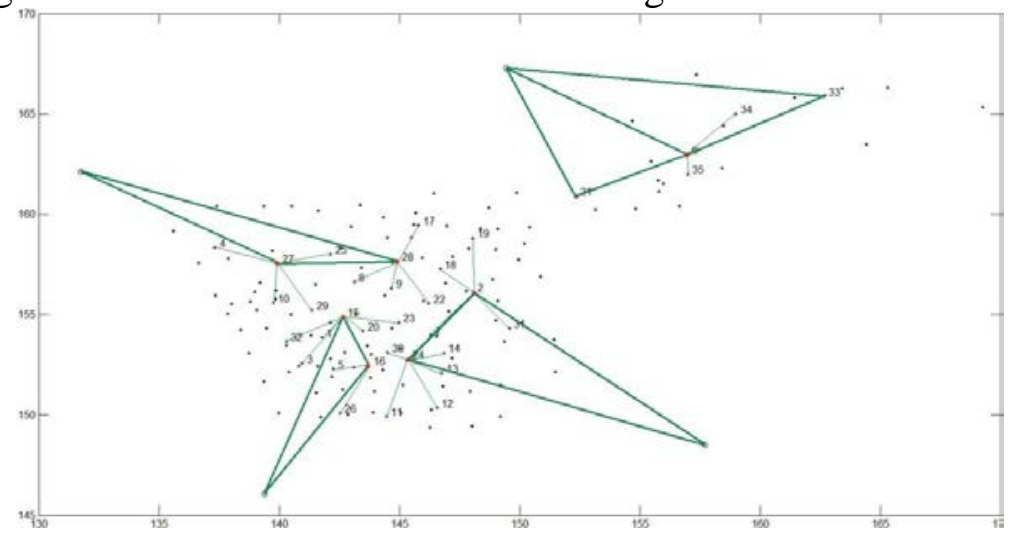

Fig. 4 Underground channel network

In the underground channel network, the coordinate position of the network nodes is shown in table 1.

Table 1 Network node coordinates

\begin{tabular}{cccccccccccc}
\hline Numble & Node & X/KM & Y/KM & Numble & Node & X/KM & Y/KM & Numble & Node & X/KM & Y/KM \\
\hline 2 & First & 148.076 & 156.132 & 5 & Secon & 142.257 & 152.293 & 20 & Secon & 143.485 & 154.183 \\
& & 4 & 7 & & $d$ & 2 & 9 & & d & 0 & 3 \\
6 & First & 157.000 & 163.000 & 7 & Secon & 146.307 & 153.965 & 22 & Secon & 146.233 & 155.539 \\
& & 0 & 0 & & d & 6 & 4 & & $\mathrm{~d}$ & 5 & 2 \\
15 & First & 142.660 & 154.903 & 8 & Secon & 143.130 & 156.632 & 23 & Secon & 144.987 & 154.599 \\
& & 3 & 0 & & $\mathrm{~d}$ & 8 & 0 & & $\mathrm{~d}$ & 1 & 4 \\
16 & First & 143.734 & 152.446 & 9 & Secon & 144.675 & 156.311 & 25 & Secon & 142.145 & 158.021 \\
& & 6 & 3 & & $\mathrm{~d}$ & 8 & 7 & & $\mathrm{~d}$ & 0 & 3 \\
21 & First & 152.337 & 160.924 & 10 & Secon & 139.760 & 155.573 & 26 & Secon & 142.533 & 150.082 \\
& & 7 & 1 & & $\mathrm{~d}$ & 2 & 2 & & $\mathrm{~d}$ & 1 & 3 \\
24 & First & 145.360 & 152.774 & \multirow{2}{*}{11} & Secon & 144.474 & 149.933 & 29 & Secon & 141.369 & 155.219 \\
& & 2 & 9 & & $\mathrm{~d}$ & 5 & 6 & & $\mathrm{~d}$ & 9 & 1 \\
27 & First & 139.942 & 157.569 & 12 & Secon & 146.562 & 150.354 & 30 & Secon & 144.505 & 153.119 \\
& & 6 & 7 & & $\mathrm{~d}$ & 8 & 8 & & $\mathrm{~d}$ & 2 & 9 \\
\hline
\end{tabular}




\begin{tabular}{|c|c|c|c|c|c|c|c|c|c|c|c|}
\hline 28 & First & $\begin{array}{c}144.913 \\
0\end{array}$ & $\begin{array}{c}157.653 \\
9\end{array}$ & 13 & $\begin{array}{c}\text { Secon } \\
\text { d }\end{array}$ & $\begin{array}{c}146.769 \\
8\end{array}$ & $\begin{array}{c}152.085 \\
2\end{array}$ & 31 & $\begin{array}{c}\text { Secon } \\
\text { d }\end{array}$ & $\begin{array}{c}149.580 \\
0\end{array}$ & $\begin{array}{c}154.320 \\
0\end{array}$ \\
\hline 33 & First & $\begin{array}{c}162.650 \\
0\end{array}$ & $\begin{array}{c}165.890 \\
0\end{array}$ & 14 & $\begin{array}{c}\text { Secon } \\
\text { d }\end{array}$ & $\begin{array}{c}146.860 \\
8\end{array}$ & $\begin{array}{c}153.094 \\
6\end{array}$ & 32 & $\begin{array}{c}\text { Secon } \\
\text { d }\end{array}$ & $\begin{array}{c}140.305 \\
1\end{array}$ & $\begin{array}{c}153.658 \\
1\end{array}$ \\
\hline 1 & $\begin{array}{c}\text { Secon } \\
\text { d }\end{array}$ & $\begin{array}{c}141.779 \\
2\end{array}$ & $\begin{array}{c}153.865 \\
0\end{array}$ & 17 & $\begin{array}{c}\text { Secon } \\
\text { d }\end{array}$ & $\begin{array}{c}146.795 \\
4\end{array}$ & $\begin{array}{c}159.457 \\
7\end{array}$ & 34 & $\begin{array}{c}\text { Secon } \\
\text { d }\end{array}$ & $\begin{array}{c}159.000 \\
0\end{array}$ & $\begin{array}{c}165.000 \\
0\end{array}$ \\
\hline 3 & $\begin{array}{c}\text { Secon } \\
\mathrm{d}\end{array}$ & $\begin{array}{c}140.956 \\
2\end{array}$ & $\begin{array}{c}152.565 \\
0\end{array}$ & 18 & $\begin{array}{c}\text { Secon } \\
\text { d }\end{array}$ & $\begin{array}{c}146.703 \\
1\end{array}$ & $\begin{array}{c}157.277 \\
7\end{array}$ & 35 & $\begin{array}{c}\text { Secon } \\
\text { d }\end{array}$ & $\begin{array}{c}157.000 \\
0\end{array}$ & $\begin{array}{c}162.000 \\
0\end{array}$ \\
\hline 4 & $\begin{array}{c}\text { Secon } \\
\text { d }\end{array}$ & $\begin{array}{c}137.328 \\
5\end{array}$ & $\begin{array}{c}158.351 \\
7\end{array}$ & 19 & $\begin{array}{c}\text { Secon } \\
\text { d }\end{array}$ & $\begin{array}{c}148.056 \\
3\end{array}$ & $\begin{array}{c}158.813 \\
5\end{array}$ & & & & \\
\hline
\end{tabular}

The number of network channels is 40 , of which, the number of network channels connected to the first level node and logistics Park is 9, the number of network channels connected by the first level node and the first level node is 5 , the number of network channels connected by the first level node and the level two node is 26, network channel specific connectivity as shown in table 2.

Table 2 The actual flow of the channel

\begin{tabular}{|c|c|c|c|c|c|}
\hline Channel & Flow/ton & Channel & Flow/ton & Channel & Flow/ton \\
\hline Logistics Park 1-Node 15 & $\begin{array}{l}1570.26 \\
5\end{array}$ & Node 2-Node 7 & 194.002 & Node 24-Node 12 & 542.280 \\
\hline Logistics Park 1-Node 16 & $\begin{array}{l}2415.40 \\
7\end{array}$ & Node 2-Node 18 & 576.423 & Node 24-Node 13 & 441.352 \\
\hline Logistics Park 2-Node 2 & $\begin{array}{l}2223.76 \\
9\end{array}$ & Node 2-Node 19 & 352.041 & Node 24-Node 14 & 404.511 \\
\hline Logistics Park 2-Node 24 & $\begin{array}{l}2326.08 \\
1\end{array}$ & Node 2-Node 31 & $\begin{array}{l}1001.31 \\
7\end{array}$ & Node 24-Node 30 & 763.582 \\
\hline Logistics Park 3-Node 6 & $\begin{array}{l}2823.53 \\
8\end{array}$ & Node 6-Node 34 & $\begin{array}{l}1884.19 \\
6\end{array}$ & Node 27-Node 4 & 112.912 \\
\hline Logistics Park 3-Node 21 & $\begin{array}{l}2983.10 \\
4\end{array}$ & Node 6-Node 35 & 939.342 & Node 27-Node 10 & 581.423 \\
\hline Logistics Park 3-Node 33 & $\begin{array}{l}2995.51 \\
9\end{array}$ & Node 15-Node 1 & 379.803 & Node 27-Node 25 & 253.485 \\
\hline Logistics Park 4-Node 27 & $\begin{array}{l}2775.24 \\
8\end{array}$ & Node 15 -Node 3 & 259.617 & Node 27-Node 29 & $\begin{array}{l}1757.31 \\
9\end{array}$ \\
\hline Logistics Park 4-Node 28 & $\begin{array}{l}2529.32 \\
5\end{array}$ & Node 15 -Node 20 & 598.101 & Node 28-Node 8 & 155.412 \\
\hline Node 2-Node 24 & 99.986 & Node 15 -Node 23 & 101.659 & Node 28-Node 9 & 820.285 \\
\hline Node 15 -Node 16 & 130.854 & Node 15-Node 32 & 100.230 & Node 28-Node 17 & 224.871 \\
\hline Node 21-Node 35 & $\begin{array}{l}1080.20 \\
1\end{array}$ & Node 16-Node 5 & 772.187 & Node 28-Node 22 & 462.005 \\
\hline $\begin{array}{l}\text { Node } 27 \text {-Node } 28 \\
\text { Node } 33 \text {-Node } 35\end{array}$ & $\begin{array}{l}70.109 \\
1782.56 \\
8\end{array}$ & $\begin{array}{l}\text { Node } 16 \text {-Node } 26 \\
\text { Node } 24 \text {-Node } 11\end{array}$ & $\begin{array}{l}1643.22 \\
174.356\end{array}$ & & \\
\hline
\end{tabular}

\section{References}

[1] Liao Хихи. Research on the location of logistics distribution center in e-commerce environment [D]. Xiamen University, 2007.

[2] Rao Liangliang. Research on the location model of regional logistics distribution center based on clustering ant colony algorithm [D]. Jiangxi University of Finance and Economics, 2012.

[3] Chen Si. Research on regional logistics planning method based on diversity of logistics demand [D]. Southwest Jiaotong University, 2013.

[4] Liu Lingrui. Research on node layout planning of regional Logistics network [D]. Changan University, 2011.

[5] Zheng bin. Research on location and route optimization of regional Logistics Network Center [D]. Dalian Maritime 
University, 2011.

[6] Wang Dan. Research on the location of logistics distribution Center [D]. Dalian Maritime University, 2007.

[7] Li Yanlai. Research on the complexity and optimization design of logistics network structure [D]. Beijing Jiaotong University, 2011.

[8] Tan Xixi. Research on optimal allocation of backbone transportation network resources of Axis-spoke express [D]. Beijing Jiaotong University, 2016.

[9] Chen Zhanbo, Huang Xiaozhou. Improved grey relational degree evaluation method for location selection of logistics distribution Center [J]. Statistics and decision making, 2015 (3): 52-55.

[10] Yu Rong, Chen Jiafeng, Gongya, et. Research on the location of logistics distribution center based on fuzzy comprehensive evaluation--a case study of Taixing Logistics in Jiangsu Province [J]. Land and resources science and technology management, 2015, 32 (4): 93-100. 